\title{
Measurement of scapula upward rotation: a reliable clinical procedure
}

\author{
L Watson, S M Balster, C Finch, R Dalziel
}

Br J Sports Med 2005;39:599-603. doi: 10.1136/bjsm.2004.013243

See end of article for authors' affiliations

Correspondence to: Ms Watson, Lifecare

Prahran Sports Medicine Centre, Level 1, 316 Malvern Road, Prahran,

Victoria 3181, Australia;

Prahran@lifecare.com.au

Accepted

10 November 2004
Background: It is important to deal with the scapula when developing rehabilitation strategies for the shoulder complex. This requires clinical measurement tools that are readily available and easy to apply and which provide a reliable evaluation of scapula motion.

Aim: To determine the reliability of the Plurimeter- $V$ gravity inclinometer for the measurement of scapular upward rotation positions during humeral elevation in coronal abduction in a group of patients with shoulder pathology.

Method: Twenty six patients were assessed in two repeat tests within a single testing session. Patients exhibiting a wide spectrum of shoulder pathology were selected. The angle of scapular upward rotation was measured during total shoulder abduction. The measurement protocol was performed twice during a single testing session by a single tester. Results of the two tests were compared and the reliability assessed by intraclass correlation coefficients (ICCs).

Results: There was no significant difference in the scapula measurements taken during the two tests at each testing position. Overall, there was very good intrarater reliability $(I C C=0.88)$. The ICC ranged from 0.81 (at $135^{\circ}$ ) to 0.94 (at both resting and end of total shoulder abduction range).

Conclusion: The Plurimeter- $V$ gravity inclinometer can be used effectively and reliably for measuring upward rotation of the scapula in all ranges of shoulder abduction in the coronal plane.
C ontribution from the scapula has long been considered essential for normal shoulder function. ${ }^{1}$ Alterations in scapula rest position ${ }^{2}$ and motion ${ }^{3-8}$ have been observed in patients with shoulder pathology. Abnormal scapulothoracic mechanics have also been implicated in the development of shoulder pathologies such as glenohumeral joint instability, impingement, and rotator cuff tears..$^{5-7} 910$ These factors highlight the need to focus on the scapula, as well as the glenohumeral joint, when developing rehabilitation strategies for the shoulder complex.

Early studies of scapulohumeral rhythm ${ }^{11-13}$ used two dimensional analysis and therefore were unable to observe three dimensional motion occurring within the shoulder complex, possibly leading to inaccurate analysis and measurement of scapula motion. ${ }^{14-17}$ Three dimensional scapula motion is known to involve rotation around several axes, ${ }^{16}$ and a variety of terms have been used to define this motion. ${ }^{18-21}$ In this study, scapula motions are defined as: upward rotation/ downward rotation (rotation about an axis perpendicular to the plane of the scapula); anterior/posterior tilt (rotation around an axis perpendicular to the spine of the scapula); internal/external rotation (rotation around an axis running vertically superior, inferior from the medial end of the spine of the scapula)..$^{15} 161922$ Scapula position and motion can also be described in terms of superior/inferior shift ${ }^{22}$ and medial/ lateral shift on the thorax. ${ }^{5622}$

Although three dimensional motion analyses may be optimal for assessing scapulohumeral kinematics, very few of these methods can be easily applied in the clinical setting, nor are they readily available from either a cost or practical perspective. ${ }^{316171923}$ Nonetheless, the onus remains on the clinician to try to objectively assess both scapula resting position and dynamic motion, as this is part of the requirement of standard physiotherapy assessment of the shoulder complex. ${ }^{615}$ In addition, the aim of many shoulder rehabilitation programmes is to correct or change aberrant scapulothoracic mechanics. This assumes that the clinician can reliably assess the scapula motion in the first place. If physiotherapists want to show that their rehabilitation strategies are effective, they must develop tools that reliably evaluate the clinical outcomes or changes that they are trying to achieve and can be easily applied in a clinical setting.

Several authors have analysed various simple clinical methods of assessing scapula motion. ${ }^{562425}$ Most have examined scapula resting position and have found good to excellent intrarater and inter-rater reliability. ${ }^{524}{ }^{25}$ Very few have analysed scapula motion through the range of humeral elevation. One study found that the universal goniometer appeared to be unreliable when applied to assessment of scapula upward rotation. ${ }^{26}$ Research on the assessment of scapula upward rotation has analysed not only the intrarater reliability of using a "modified" digital inclinometer, but also validated their inclinometer (two dimensional measurement) against a three dimensional electromagnetic tracking system. ${ }^{17} 2327$ Johnson et a l $^{17}$ showed not only excellent intrarater reliability in assessing scapular upward rotation in four static positions of humeral elevation, but also good to excellent concurrent validity between the inclinometer and the three dimensional magnetic tracking device. This research ${ }^{15}{ }^{17} 23$ is extremely valuable as it shows that a simple clinical assessment tool, such as a "modified" inclinometer, may be a valid and reliable measurement device for assessing upward rotation of the scapula both at rest and during humeral elevation.

Previous research has shown excellent intra-therapist and inter-therapist reliability for the Plurimeter- $V$ inclinometer in the measurement of glenohumeral joint range of motion. ${ }^{28}$ The Plurimeter- $\mathrm{V}$ was already accessible in the clinic, was cost effective, required no modification, and clinicians were already familiar with its use. In a complete analysis of scapula position, all of the scapula motions would be

Abbreviations: ICC, intraclass correlation coefficient; TSA, total shoulder abduction 


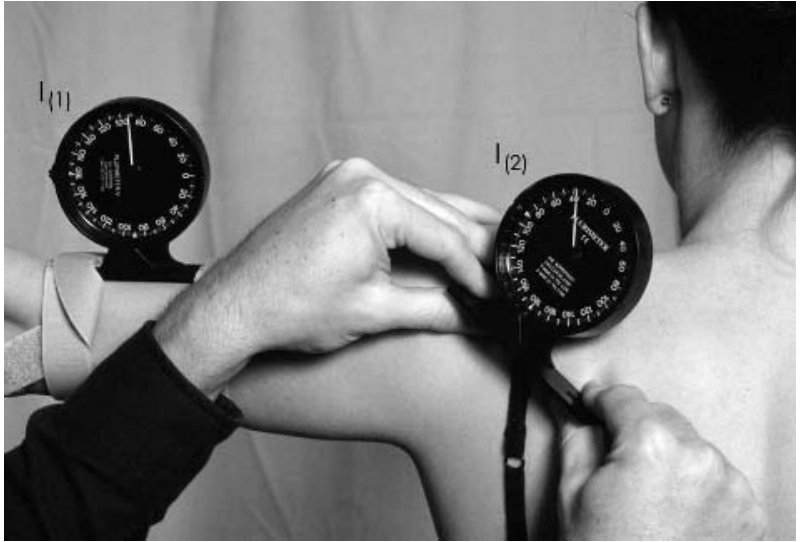

Figure 1 Positioning of the two Plurimeter-V gravity inclinometers during data collection. One inclinometer $\left(I_{1}\right)$, Velcro taped to the distal humerus, was used to determine total shoulder abduction. The other inclinometer $\left(\mathrm{I}_{2}\right)$ was manually positioned along the spine to the scapula to measure the degree of scapula upward rotation. The patient has given permission for the publication of this figure.

examined, not just upward rotation of the scapula. Unfortunately, the Plurimeter- $\mathrm{V}$ is not able to measure other rotations of the scapula, and further research is required to develop clinical measures that can accurately and objectively evaluate these motions. Hence, the primary aim of this paper was to determine if the Plurimeter- $V$ gravity referenced inclinometer was reliable for measuring scapular upward rotation positions during total shoulder abduction (TSA) in a group of patients with shoulder pathology. It is acknowledged that this is only a partial assessment of scapula motion, but, given the paucity of objective measures currently available to clinicians, we felt that it was important to document the reliability of measurement of at least one of the rotations of the scapula.

\section{METHODS \\ Subjects}

Twenty six patients (11 male, 15 female) with a history of shoulder pathology were recruited from a sample of convenience. The mean (SEM) age was 29 (2.5) years. Twenty five patients were right hand dominant, and one was left. Four had bilateral shoulder conditions, and 22 had unilateral shoulder pain. Twenty had their right shoulder measured, and six had their left shoulder measured, resulting in 19 dominant and seven non-dominant shoulders being measured. The symptomatic shoulder was selected for testing in patients with unilateral shoulder pain, and the most problematic shoulder was selected for testing in patients with bilateral shoulder pain.
Patients were recruited from the Lifecare Prahran Sports Medicine Centre and Melbourne Orthopaedic Group and were selected to represent a wide spectrum of shoulder pathology. Inclusion criteria for patients with shoulder pathology were: pain and/or dysfunction in one or both shoulders; a diagnosis of shoulder pathology determined by an orthopaedic surgeon; $>140^{\circ}$ of humeral elevation in the coronal plane (abduction) as measured by the Plurimeter-V inclinometer (using a previously developed protocol ${ }^{28}$ ). Exclusion criteria included: congenital defect of the scapula or thorax; history of trauma, fracture, or surgery to the scapula, rib cage, or thoracic spine; any evidence of peripheral or central nerve lesion, such as long thoracic nerve or accessory nerve; known neuromuscular disorders; $<140^{\circ}$ of combined TSA; an irritable shoulder condition, defined as one with shoulder pain increasing appreciably with repeated motion and being slow to settle once aggravated.

The study occurred over a six week period. All patients were interviewed and examined for inclusion/exclusion criteria by one author (LW), and an explanation of the testing procedure was given including informed consent and a copy of the pain rating system to be used in the trial.

Patients had had their symptoms for a mean (SEM) of 45 (11.3) months. Three had a history of shoulder trauma, and four had a history of shoulder surgery. Twelve were involved in manual occupations, and 14 were involved in sedentary employment. Fifteen patients were participated in recreational sports (tennis, swimming, hockey, motocross, Australian rules football, javelin, netball, triathlon, basketball), and two of these were involved in high level sport (tennis, swimming). Patients were specifically selected to represent both sporting and non-sporting populations as either may present clinically with shoulder pain, and it was felt that it was important that the results could be generalised to either population.

Patients were diagnosed as having multidirectional instability ( $\mathrm{n}=7$ ), shoulder instability (3), post shoulder dislocation (2), shoulder overuse injury (3), thoracic outlet syndrome (3), shoulder degeneration (2), scapular dyskinesia (4), SLAP lesion (1), and shoulder impingement (1).

All patients signed a written consent form, and the Melbourne Orthopaedic Group Ethics Committee approved the use of human subjects for this study.

\section{Instrumentation}

Two Plurimeter-V gravity referenced inclinometers (fig 1) were used for the study. The gravity referenced inclinometer is calibrated on the basis of gravity, and therefore the starting position of the movement to be measured is fixed, minimising placement error. The Plurimeter- $\mathrm{V}$ is accurate to within $1^{\circ}$ task. ${ }^{28}$ One inclinometer $\left(I_{1}\right)$ was used to measure the five humeral elevation positions analysed in the study, and a second inclinometer $\left(\mathrm{I}_{2}\right)$ was used to measure upward rotation of the scapula.

Table 1 Summary of the sequential clinical measurement procedure

\begin{tabular}{ll}
\hline Task & Measurement \\
\hline $\begin{array}{l}\text { Patient externally rotates their arm by their side } \\
\text { Assessor fixes inclinometer }\left(\mathrm{I}_{1}\right) \text { to lateral distal humeral } \\
\text { shaft using Velcro straps }\end{array}$ & $\begin{array}{l}\text { No measurement taken } \\
\text { Measure resting abduction angle from } \mathrm{I}_{1} \text { (relative to } \\
\text { vertical) }\end{array}$ \\
$\begin{array}{l}\text { Assessor palpates the spine of the scapula and aligns } \\
\text { the inclinometer }\left(\mathrm{I}_{2}\right) \text { along the scapula spine }\end{array}$ & $\begin{array}{l}\text { Measure resting scapula spine angle from } \mathrm{I}_{2} \text { (relative to } \\
\text { horizontal) }\end{array}$ \\
$\begin{array}{l}\text { Patient abducts their arm and is instructed to stop at } \\
\text { each of } 45^{\circ}, 90^{\circ}, 135^{\circ} \text { and end of range abduction }\end{array}$ & $\begin{array}{l}\text { Measure scapula spine angle at each abduction } \\
\text { position using } \mathrm{I}_{2} \text { (relative to horizontal) } \\
\text { Measure end of range abduction angle using } \mathrm{I}_{1} \\
\text { (relative to vertical) }\end{array}$ \\
\hline
\end{tabular}


Table 2 Descriptive statistics, standard error of measurement (SEM), and intraclass correlation coefficient (ICC) of the scapula measurements at each position over two trials

\begin{tabular}{|c|c|c|c|c|c|}
\hline TSA position & Trial & Mean (SD) & Range & SEM & ICC $(95 \% \mathrm{Cl})$ \\
\hline At rest & 1 & $3.58(7.23)$ & -15 to 20 & 1.7 & $0.94(0.90$ to 0.99$)$ \\
\hline (average $10.2^{\circ}$ ) & 2 & $3.92(7.06)$ & -12 to 16 & & \\
\hline \multirow{2}{*}{ At $45^{\circ}$} & 1 & $13.19(11.09)$ & -10 to 36 & 4.0 & 0.88 (0.79 to 0.97$)$ \\
\hline & 2 & $12.85(11.40)$ & -6 to 39 & & \\
\hline \multirow[t]{2}{*}{ At $90^{\circ}$} & 1 & 28.35 (13.01) & 0 to 56 & 3.8 & $0.90(0.82$ to 0.97$)$ \\
\hline & 2 & $28.12(10.46)$ & 3 to 55 & & \\
\hline At $135^{\circ}$ & $\begin{array}{l}1 \\
2\end{array}$ & $\begin{array}{l}41.46(13.13) \\
44.77(10.45)\end{array}$ & $\begin{array}{l}13 \text { to } 65 \\
17 \text { to } 62\end{array}$ & 5.2 & 0.81 (0.67 to 0.94$)$ \\
\hline At end of range & 1 & $56.58(12.66)$ & 27 to 75 & 2.9 & $0.94(0.89$ to 0.98$)$ \\
\hline (average $174.6^{\circ}$ ) & 2 & $55.19(11.13)$ & 25 to 75 & & \\
\hline Overall & & & & & $0.88(0.73 \text { to } 1.00)^{*}$ \\
\hline
\end{tabular}

*The $95 \%$ confidence interval calculated is the one sided, lower limit confidence interval. TSA, Total shoulder abduction.

\section{Standardised testing protocol}

All patients were assessed in a relaxed, balanced standing position with their feet positioned shoulder width apart.

Scapular upward rotation was measured during TSA using a standardised reliable protocol..$^{28}$ Total shoulder abduction involves glenohumeral and scapular motion within the coronal plane. This was measured with full elbow extension, neutral wrist flexion/extension, and with the thumb leading to ensure consistent vertical alignment of the inclinometer. Table 1 summarises the testing procedure adopted.

The Plurimeter that was used to measure TSA angles $\left(I_{1}\right)$ was Velcro taped perpendicular to the shaft of the humerus, just above the lateral humeral epicondyle (fig l). The Plurimeter dial was rotated such that a dial reading of $0^{\circ}$ corresponded to vertical.

The actual resting position/angle of each patient's humerus, relative to vertical, was recorded. Patients were then asked to move both arms into abduction and asked to stop at $45^{\circ}, 90^{\circ}$, and $135^{\circ}$ and at their maximum achievable range (which was also documented). At each of these abduction positions, the degree of upward rotation of the scapula was measured using a second inclinometer $\left(\mathrm{I}_{2}\right)$. This was achieved by manually aligning the base of the inclinometer along the spine of the scapula. At each measurement, the patient was asked if any pain was present, and this was graded and recorded if it was present according to the five point category system as outlined by Green. ${ }^{28}$

\section{Reliability testing}

All patients were measured by one of the authors (SB) who had previous experience using the Plurimeter- $V$ inclinometer. Two rounds of testing were conducted on each patient within a single testing session and were recorded on separate standardised data collection sheets. The patients were led through a series of warm up exercises involving flexion and abduction in standing. The humeral inclinometer was fitted, and it was checked that it was centred to 0 and vertically aligned. The patient was then instructed to assume a relaxed stance and externally rotate their arm for measurement of the resting humeral angle and the resting angle of scapula upward rotation. The patient was then instructed to abduct their arm to $45^{\circ}, 90^{\circ}, 135^{\circ}$ and end of range TSA. At each of these abduction positions, the static angle of the scapula upward rotation was measured. Each measurement took about 10 seconds. No randomisation of order of testing was performed. No communication about the measurements occurred between the patient and the examiner apart from noting whether or not any pain was present and, if so, its level of severity at each measurement position. The humeral inclinometer was then removed, and the patient was instructed to lie down for half an hour and given some light reading. After 30 minutes, round 2 of the testing was then performed according to the exact protocol of round 1 .

\section{Data analysis}

The data were analysed, and statistical evaluation was carried out using Stata Statistical Software, release 7.0. Descriptive statistics of the scapula measurements for each humeral abduction position were produced for both trials. The reliability of the testing procedure, both overall and at each position, was assessed by intraclass correlation coefficients (ICCs). ${ }^{29}$ The ICC was determined from a variance components analysis, which assumes a random effect for patient in an analysis of variance model.

\section{RESULTS}

Table 2 shows the descriptive statistics of the scapula measurements at each position. As expected, the mean angle of scapula upward rotation increased with increasing TSA angle. The standard error of measurement was calculated for each stage of measurement and found to be greatest at the $135^{\circ}$ abduction position and least at the resting abduction position. There were no significant differences in mean scapula upward rotation angles between testing rounds 1 or 2 for any abduction position (one way analysis of variance, $\mathrm{p}<0.001$ for all testing positions). The mean (SEM) angle of the patient's humerus was $10.2(0.91)^{\circ}$ at rest and 174.6 (1.6) ${ }^{\circ}$ at the end of range. The Plurimeter- $\mathrm{V}$ inclinometer showed good to excellent intrarater reliability within a single session at all positions of TSA using an ICC analysis. The reliability of the measurement was highest at rest and at the end of TSA range.

\section{DISCUSSION}

The reliability of assessing scapular rest positions with simple, clinically available measurement methods has been dealt with in the literature. ${ }^{6} 172425$ Our results compare well with previous research with respect to intrarater reliability. Greenfield et $a l^{25}$ achieved an ICC of 0.97 , the intrarater correlation coefficient of Johnson et al $^{17}$ ranged from 0.89 to 0.96 , and DiVita $e t$ al $^{24}$ quoted an ICC of 0.94 .

Although our study showed excellent reliability of measurement of scapula motion overall (ICC $=0.88$ ), it is important to be aware that there was some variability in the levels of reliability shown, relative to the range of abduction being assessed. The greatest reliability was seen in measurement of the scapula at rest and at maximum range of abduction, and the least reliability was seen at $135^{\circ}$ of abduction. This is reflected in the through abduction range variation of the standard error of measurement. This may be because clinically $135^{\circ}$ of abduction is often the most pain provocative position. Unfortunately, although we recorded 
each subject's level of pain during TSA, only four subjects experienced pain. Therefore further statistical analysis with more patients is required to examine this fully. Nonetheless, it is important that the therapist takes extra care when measuring this part of the range. The standard error of measurement data indicate that, from a clinical perspective, a change of more than $5^{\circ}$ is required to demonstrate a change in upward rotation of the scapula.

A major difference between our study and previous work is that other studies ${ }^{11} 121617193031$ assume that the scapula resting position is at $0^{\circ}$ of humeral elevation. In our study, we measured the actual angle of the humerus at rest and found that it averaged $10.2(0.91)^{\circ}$ of abduction and not $0^{\circ}$. Furthermore, comparison across studies is difficult because of methodological differences, including actual humeral resting position, specific arm motion studied, static versus dynamic testing, trunk position, and type of instrumentation. ${ }^{15}$

The study of Johnson et $a l^{17}$ is the best available for comparison. They found a mean (SEM) of $2.8(6.1)^{\circ}$ for scapula upward rotation at actual humeral resting position using a modified digital inclinometer. ${ }^{17}$ This compares favourably with our study, which found a mean (SEM) of $3.75(7.15)^{\circ}$ upward rotation for average resting position of $10.2^{\circ}$ TSA. Most of the other studies ${ }^{24}{ }^{32-34}$ used unimpaired subjects with no history of shoulder pathology. This limits the generalisability of their results to reliability of measurement of scapular position in the asymptomatic patient population only. Our study, and that of Johnson et al, ${ }^{17}$ confirmed that an inclinometer could be used to reliably measure scapular upward rotation in patients with shoulder pathology, which is essential for clinical application.

However, it is important to emphasise that only scapular upward rotation was examined in this study. This is a significant limitation, as other motions of the scapula are equally as important to examine. Unfortunately the inclinometer in its current form is not able to measure these other motions. To our knowledge, no other simple, cheap, readily available objective measurement method has been documented to measure these other scapula motions. Kibler ${ }^{5}$ has demonstrated an objective measurement technique for lateral/medial shift of the scapula. Scapula asymmetry has been associated with shoulder pathology by numerous authors. ${ }^{4-7}$ Various abnormalities in scapula motion have been implicated in the development of clinical symptoms. ${ }^{5-7} 22$ The relative importance of any one specific scapula motion being altered and its impact on coupled glenohumeral motions has not been fully assessed. This is partly because objective measurement devices for each of these motions have not been readily available. Now that the reliability of the Pluirmeter- $V$ has been established, further research will be able to determine how often upward/downward rotation is affected and how important it is to rehabilitate in injured patients. It is suggested that it be used in conjunction with other objective measures, such as the lateral scapula slide test. ${ }^{5}$ Until a clinical tool is available that can accurately measure all the scapula motions, the clinician should keep in mind that measuring upward rotation alone may not provide all the answers. An absence of noteworthy findings in upward rotation measure does not exclude major alterations in other scapula motions. Measurement of changes in upward rotation may not reflect changes in other planes of motion and may introduce a margin of error in measurement findings. However, it is essential that objective documentation of scapula motion occurs, even if it is only partial. Only with further research on greater patient numbers will the strengths and limitations of this measure be fully established.

TSA in the coronal plane was assessed in this study, whereas in other studies ${ }^{35-1723}$ humeral elevation has been
What is already known on this topic

Measurement of scapula rest position and motion in the clinic is confounded by its multiplanar kinematics. Initial research described methods to measure scapula rest position. Recent research has developed a reliable clinical tool to measure scapula motion during arm elevation and has validated this method against a three dimensional electromagnetic tracking device.

\section{What this study adds}

This study indicates that the Plurimeter- $\mathrm{V}$ inclinometer can be used to reliably measure the angle of scapula upward rotation during total shoulder abduction. This gives physiotherapists a cheap, easy tool for assessing scapula upward rotation and evaluating it in terms of shoulder pathology, rehabilitation objectives, and clinical outcomes.

assessed by measurement in the plane of the scapula. Elevation in the plane of the scapula has been suggested to be the most functional plane for arm elevation, ${ }^{35-37}$ and this may indeed be true. However, this does not mean that it is not also essential to examine other planes of elevation. A standard physiotherapy examination of the shoulder complex includes assessment of humeral elevation through both flexion and abduction. ${ }^{5637}$ As Johnson et al ${ }^{17}$ had already demonstrated both the reliability and validity of the use of their modified digital inclinometer for measuring scapular upward rotation in elevation in the plane of the scapula, it was felt that there was a need to assess whether or not a simple, clinically available device could also measure scapular upward rotation in TSA. Clinically, abduction is often more pain provocative and dysfunctional than other shoulder motions, especially in patients with impingement and rotator cuff pathology, ${ }^{46-41}$ and several other authors have highlighted the fact that asymmetry of the scapulae is commonly seen in abduction. ${ }^{46}$ Abduction is also a very important movement to consider if the clinician is trying to assess the scapula position in higher levels of functional tasks, such as throwing, as the scapula must move into the coronal plane for these motions to occur.

We examined the full range of abduction $\left(180^{\circ}\right.$ or maximum range available) as we wanted to establish the reliability of the Plurimeter-V inclinometer over as large a range as possible to ensure that it could be applied to as broad a patient population as possible. Some studies have only considered scapula motion up to $120^{\circ}$ of elevation (in the plane of the scapula). ${ }^{15-17}$ There is evidence ${ }^{15}$ that a considerable amount of upward rotation of the scapula may occur after $120^{\circ}$ of elevation, hence we felt that it was important to examine the scapula throughout TSA. It is of interest to note that not all patients can achieve $180^{\circ}$ of abduction. The mean (SEM) end of range measurement was 174.6 (1.6 $)^{\circ}$. Shoulder pathology often limits scapulothoracic motion, and this is the most likely cause of any limitation of TSA movement. However, just as the resting position of the humerus is rarely $0^{\circ}$ abduction, one must not assume that end of range of motion in TSA is always necessarily $180^{\circ}$.

A limitation of this study may be that the testing order of the motions was not randomised. We originally decided not to randomise the testing order, as we wanted to test the reliability of the assessment procedure as it would actually be used in a clinical setting. However, a second patient series to 
examine the effect of randomisation on the order of testing is underway, as this may strengthen our findings.

Finally, from a clinical perspective, it would be inaccurate to extrapolate our scapula upward rotation measurements to specific patients. Our patient population was chosen to represent a wide cross section of sport and non-sport related shoulder pathologies. Therefore the mean angles of scapula upward rotation obtained will not accurately reflect that of a specific pathological group. This is reflected in the wide range in $(\min -\max )$ scapula upward rotation angles observed. Further research on specific patients groups is needed to establish typical reference ranges of scapula upward rotation.

In summary, we have shown that the Plurimeter-V can be used effectively and reliably for measuring upward rotation of the scapula in all ranges of TSA in the coronal plane.

\section{ACKNOWLEDGEMENTS}

We thank Tim Wrigley, Senior Research Officer, Centre for Rehabilitation, Exercise and Sport Science, Victoria University, for assistance in developing the method and protocol used in this study, and Vasuki Prabaharan from Monash University, Department of Epidemiology and Preventive Medicine, for completing the statistical analysis of the data.

\section{Authors' affiliations}

L Watson, S M Balster, LifeCare, Prahran Sports Medicine Centre, Prahran, Victoria, Australia

C Finch, NSW Injury Risk Management Research Centre, University of New South Wales, Sydney, Australia

R Dalziel, Melbourne Orthopaedic Group, Windsor, Victoria, Australia Competing interests: none declared

Permission has been obtained from the patient for the publication of fig 1.

\section{REFERENCES}

1 Inman V, Saunders M, Abbott L. Observations on the function of the shoulder joint. J Bone Joint Surg [Am] 1944;26:1-30.

2 Solem-Bertoft E, Thuomas K, Westerberg C. The influence of scapular retraction and protraction on the width of the subacromial space. Clin Orthop 1993;296:99-103.

3 Lukasiewicz A, McClure P, Michener L, et al. Comparison of three dimensional scapular position and orientation between subjects with and without shoulder impingement syndrome. J Orthop Sports Phys Ther 1999;29:574-86.

4 Warner J, Micheli L, Arslanian L, et al. Scapulothoracic motion in normal shoulders and shoulders with glenohumeral instability and impingement syndrome. A study using Moire topographic analysis. Clin Orthop 1992;285:191-9.

5 Kibler $\mathrm{W}$. The role of the scapula in the overhead throwing motion. Contemp Orthop 1991;22:525-32.

6 Kibler W. Evaluation and diagnosis of scapulothoracic problems in the athlete. Sports Med Arthrosc Rev 2000;8:192-202.

7 Ozaki J, Fujimoto S, Mashuhara K. Repair of chronic massive rotator cuff tears with synthetic materials. Clini Orthop 1986;202:173-83.

8 Paletta G, Warner J, Warren R, et al. Shoulder kinematics with 2-plane x-ray evaluation in patients with anterior instability or rotator cuff tearing. J Shoulder Elbow Surg 1997;6:516-27.

9 Matsen F, Lippitt S, Sidles J, et al. Practical evaluation and management of the shoulder. Philadelphia: WB Saunders, 1994.

10 Kendall H, Kendall F, Wadsworth G. Muscle testing and function. 2nd ed: Williams and Wilkins Company, 1971.

11 Poppen N, Walker P. Normal and abnormal motion of the shoulder. J Bone Joint Surg [Am] 1976;58:195-201.

12 Inman V, Saunders J, Abbott L. Observations on the function of the shoulder joint. J Bone Joint Surg [Am] 1944;26:1-30.
13 Freedman L, Munro R. Abduction of the arm in the scapular plane: scapular and glenohumeral movements. A roetenographic study. J Bone Joint Surg [Am] 1966;48:1503-10.

14 De Groot J. The scapulo-humeral rhythm: effects of 2-D roentgen projection. Clin Biomech 1999;14:63-8.

15 McClure P, Michener L, Sennelt B, et al. Direct 3-dimensional measurement of scapular kinematics during dynamic movements in vivo. J Shoulder Elbow Surg 2001;10:269-77.

16 Ludewig P, Cook T, Nawoczenski D. Three dimensional scapula orientation and muscle activity at selected positions of humeral elevation. J Orthop Sports Phys Ther 1996;24:57-65.

17 Johnson M, McClure P, Karduna A. New method to assess scapular upward rotation in subjects with shoulder pathology. J Orthop Sports Phys Ther $2001 ; 31: 81-9$.

18 Dempster W. Mechanisms of shoulder movement. Arch Phys Med Rehabil 1965;46:49-70.

19 McQuade K, Smidt G. Dynamic scapulohumeral rhythm: the effects of external resistance during elevation of the arm in the scapular plane. J Orthop Sports Phys Ther 1998;27:125-33.

20 Morrey B, An KN. Biomechanics of the shoulder. In: Matsen F, ed. The shoulder. Philadelphia: WB Saunders Company, 1990:208-45.

21 Norkin C, Levangie P. Joint structure and function: a comprehensive analysis. Philadelphia: FA Davis Company, 1992:215-16.

22 Wang CH, McClure P, Pratt NE, et al. Stretching and strengthening exercises: their effect on three-dimensional scapular kinematics. Arch Phys Med Rehabil 1999;80:923-9.

23 Karduna A, McClure P, Michener L, et al. Dynamic measurements of three dimensional scapular kinematics: a validation study. J Biomech Engineering 2001;123:184-90.

24 Diveta J, Walker M, Skibinski B. Relationship between performance of selected scapular muscles and scapular abduction in standing subjects. Phys Ther 1990;70:470-6.

25 Greenfield B, Catlin P, Coats $\mathrm{P}$, et al. Posture in patients with shoulder overuse injuries and healthy individuals. J Orthop Sports Phys Ther 1995;21:287-95.

26 Youdas J, Carey J, Garrett T, et al. Reliability of goniometric measurements of active arm elevation in the scapula plane obtained in a clinical setting. Arch Phys Med Rehabil 1994;75:1 137-44.

27 McClure P, Michener L, Sennett B, et al. Direct 3-dimensional measurement of scapular kinematics during dynamic movements in vivo. J Shoulder Elbow Surg $2001 ; 10: 269-77$.

28 Green S. A standardized protocol for measurement of range of movement of the shoulder using the Plurimeter $V$ inclinometer and assessment of its intrarater and interrater reliability. Arthritis Care and Research 1998; 11:43-52.

29 Fleiss J. Statistical methods for rates and proportions. New York: John Wiley \& Sons, 1981.

30 Bagg S, Forrest W. Electromyographic study of the scapular rotators during arm abduction in the scapular plane. Am J Phys Med Rehabil 1986;65:111-24

31 Bagg S, Forrest W. A biomechanical analysis of scapular rotation during arm abduction in the scapular plane. Am J Phys Med Rehabil 1988;67:238-45.

32 Gibson M, Goebel G, Jordan T, et al. A reliability study of the measurement techniques to determine static scapular position. J Orthop Sports Phys Ther 1995;21:100-6.

33 T'Jonck L, Lysens R, Grade G. Measurement of scapular position and rotation: a reliability study. Phsyiother Res Inter 1996;1:148-58.

34 Sobush D, Simoneau g, Dietz K, et al. The Lennie test for measuring scapular position in healthy young adult females: a reliability and validity study. $J$ Orthop Sports Phys Ther 1996;23:39-50.

35 Saha A, Das N, Chakravarty B. Studies on electromyographic changes of muscles acting on the shoulder joint complex. Calcutta Med J, 53:409-13.

36 Saha A. Theory of shoulder mechanism: descriptive and applied. Springfield, IL: Charles C Thomas, 1961.

37 Kelly B, Warren K, Speer K. The manual muscle examination for rotator cuff strength. An electromyographic investigation. Am J Sports Medicine 1996;24:581-8.

38 Hawkins R, Kennedy J. Impingement syndrome in athletes. Am J Sports Med 1980;8:151-8.

39 Kennedy J, Hawkins R, Krissoff W. Orthopaedic manifestations of swimming. Am J Sports Med 1978;6:309-22.

40 Burkhart S, Morgan C, Kibler W. Shoulder injuries in overhead athletes. The "dead arm" revisited. Clin Sports Med 2000;19:125-58.

41 Neer C. Impingement lesions. Clin Orthop 1983;173:70-7. 